\title{
Model Free Backstepping Control for Marine Power Systems
}

\author{
Sun Jianlong ${ }^{1, *}, \mathrm{Xu}$ Dezhi $^{2}$, Zha Shensen ${ }^{3}$ and $\mathrm{Ge} \mathrm{Le}^{4}$ \\ ${ }^{I}$ School of Electrical Engineering, Southeast University, Nanjing, 210096, P.R. China \\ ${ }^{2}$ Key Laboratory of Advanced Process Control For Light Industry (Ministry of Education), Institute of Automation, \\ Jiangnan University, Wuxi, 214122, P.R. China \\ ${ }^{3}$ Jiangsu Electric Power Design Institute, Nanjing, 211102, P.R. China \\ ${ }^{4}$ School of Electric Power Engineering, Nanjing Institute of Technology, 211167, Nanjing, P.R. China
}

\begin{abstract}
In order to retrain chaotic oscillation of marine power systems which are excited by periodic electromagnetism perturbation, in this paper, a novel model free backstepping control methods are designed. First, the dynamic model of marine power system is established based on the two parallel nonlinear model. Then, extended state observer (ESO) is designed to estimate the velocity signal and unknown function. Next, the model free backstepping controller is proposed based on the ESO. Finally, simulation results demonstrate the proposed model free control algorithm can quickly retrain chaotic oscillation. And it shows the proposed control method is effectiveness and potential.
\end{abstract}

Keywords: Model free control, backstepping, extended state observer, marine power systems.

\section{INTRODUCTION}

Structure of modern marine power systems has been ever more complicated, especially the emergence of highperformance ship electric propulsion applications. With the development of modern marine power system becomes more and more complex, more extreme the reliability and stability requirements are need to marine power systems. In recent years, researchers found that chaotic oscillations are occurred in marine power system during the voyage or paroxysmal bursts. Chaotic oscillations could lead to system instability, which poses a potential threat to the safe operation of the marine power grid [1-3]. At present, most the power system chaos control method is mainly focus on land-based power systems, such as adaptive control, feedback control, inverse system control [2-7]. Obviously, the marine power systems can be regarded as a special case of land-based power systems, so a large number of control methods of land-based power systems can be extended to marine power systems. However, in the actual system, the accurate value of speed signal and the model parameters are difficult to obtain, this will make a lot of model-based control algorithms difficult to be applied [8].

In control theory, backstepping is a technique which is proposed in 1990s for designing stabilizing controls of strictfeedback nonlinear dynamical systems [9]. These systems are established from multi-subsystems that emit out from an reducible subsystem that can be stabilized with some other approaches. Since this recursive structure, researchers can start the design procedure under the known stability system and "back-out" new controllers that gradually stabilized each outer subsystems. The procedure terminates while the final external control is achieved. This procedure is called as backstepping. So far, backstepping control has made many achievements, like adaptive backstepping control, adaptive sliding mode backstepping control, dynamic surface control and so on [10-12].

Recently, model free control is increasingly receiving attention in solving complex and practical problems, such as active disturbance rejection control (ADRC) [13], model free adaptive control (MFAC) [14, 15], and so on. Summary aforementioned works, the paper gives a model free backstepping control method for marine power systems. In order to suppress the chaotic marine power system oscillations, based on extended state observer (ESO), model free backstepping chaos controller is designed. This paper is organized as follows. In Section 2, a brief description for two parallel nonlinear mathematical models is given. In Section 3 , main results are given. Simulation results are presented to show the effectiveness of the proposed control technique in Section 4. Finally, some conclusions are made in Section 5.

\section{MARINE POWER SYSTEM MODELING AND PROBLEM FORMULATION}

The basic structure of the power supply network for marine power system can be expressed as Fig. (1). Where $E_{1} \angle \delta_{1}$ and $E_{2} \angle \delta_{2}$ are emf of two generators in the system, respectively. $x_{d 1}^{\prime}$ and $x_{d 2}^{\prime}$ are synchronous reactance of two generators, respectively. $x_{l}$ and $r_{l}$ are the line resistance and 


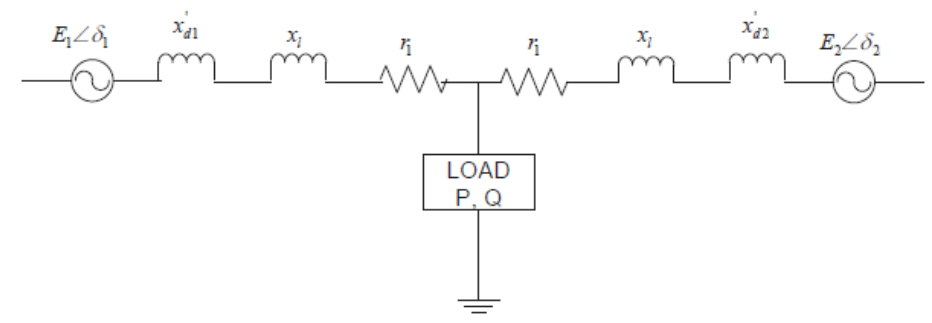

Fig. (1). Block diagram of the two-parallel model.

reactance, respectively. $P$ and $Q$ describe the system load. Because of the short-circuit in the marine power system, the line resistance is very small, which often can be neglected.

Consider same case of generator parameters, let $\delta=\delta_{1}-\delta_{2}$, and $\omega=\omega_{1}-\omega_{2}$ are relatively power angle and relative power angle velocity of the two equivalent generators. Then two machines interconnected system can be described as following form

$$
\left\{\begin{array}{l}
\frac{d \delta}{d t}=\omega \\
H \frac{d \omega}{d t}=P_{m}-D \omega-P_{e}(1+\Delta p \cos (\beta t)) \sin \delta
\end{array}\right.
$$

where $H$ and $D$ are equivalent inertia and damping, respectively. $P_{m}$ is the input mechanical power of generator, $P e$ is the electromagnetic power of system output. $\Delta p \cos (\beta t)$ is electromagnetic perturbation which is introduced to study chaotic motion for the marine power system under disturbance. Where $P_{e} \cdot \Delta p$ describes the amplitude of disturbance, and $\beta$ describes the frequency of disturbance.

Through the transformation $\tau=t \sqrt{P_{e} / H}, x_{1}(\tau)=\delta(t)$ and $x_{2}(\tau)=\sqrt{P_{e} / H} \omega(t)$. Eq. (1) can be written as

$$
\left\{\begin{array}{l}
\frac{d x_{1}}{d \tau}=x_{2} \\
\frac{d x_{2}}{d \tau}=-\sin x_{1}-\lambda x_{2}+\rho+\mu \cos (\gamma \tau) \sin x_{1}
\end{array}\right.
$$

where $\lambda=D \sqrt{P_{e} / H}, \rho=P_{m} / P_{e}, \mu=\Delta p \quad, \gamma=\beta \sqrt{P_{e} / H}$. According to transformation, we know that the system state variables $x_{1}$ and $x_{2}$ were obtained by the transformation of $\delta$ and $\omega$, which have the physical meaning of power angle error and the power angle error relative velocity between the two generators. However, if the value $\sqrt{H / P_{e}}$ is imprecise, accurate state $x_{2}(\tau)$ cannot be obtained. On the following works, a novel model free control method is proposed under only power angle $\delta(t)$ can be measured.
In order to ascertain subject for further elaboration, we define $\dot{a}=\frac{d a}{d \tau}$. In this paper, then (2) can be rewritten as $\left\{\begin{array}{l}\dot{x}_{1}=x_{2} \\ \dot{x}_{2}=-\sin x_{1}-\lambda x_{2}+\rho+\mu \cos (\gamma \tau) \sin x_{1}\end{array}\right.$

Let $x=\left[x_{1}, x_{2}\right]^{T}, f(x)=-\sin x_{1}-\lambda x_{2}+\rho+\mu \cos (\gamma \tau) \sin x_{1}$. In the above marine power system (3), when amplitude $\mu$ and frequency $\gamma$ of disturbance meet certain conditions, the chaotic motion will be occurred. In order to suppress the chaotic motion, a control input $u$ must be added to the equation of state (3), namely

$\left\{\begin{array}{l}\dot{x}_{1}=x_{2} \\ \dot{x}_{2}=f(x)+u \\ y=x_{1}\end{array}\right.$

If cannot obtain the parameters of model (3), $f(x)$ can be seen as an unknown function, and the state $x_{2}$ cannot also be measured.

\section{MAIN RESULTS}

\subsection{Extended State Observer Design}

Because we assume that only power angle $\delta=y$ can be measured for marine power system (3). So in this paper, the third-order ESO is designed, which is used to estimate the state $x_{2}$ and unknown function $f(x)$. And define unknown function $f(x)$ as an extended state $x_{3}$. Let $x_{3}=f(x)$, $\dot{x}_{3}=\varpi$, where $\rho(t)$ is a unknown function. We assume that $|\varpi(t)|<r$. Then system (4) is equivalent to

$$
\left\{\begin{array}{l}
\dot{x}_{1}=x_{2} \\
\dot{x}_{2}=x_{3}+u \\
\dot{x}_{3}=\varpi \\
y=x_{1}
\end{array}\right.
$$


In order to estimate the state $x_{2}$ and unknown function $f(x)$, we design the following third-order ESO $[13,16]$ :

$$
\left\{\begin{array}{l}
\dot{\hat{x}}_{1}=\hat{x}_{2}-l_{1} e \\
\dot{\hat{x}}_{2}=\hat{x}_{3}+u-l_{2} \mathrm{fal}\left(e, \alpha_{1}, \sigma_{1}\right) \\
\dot{\hat{x}}_{3}=-l_{3} \mathrm{fal}\left(e, \alpha_{2}, \sigma_{2}\right) \\
\hat{y}=\hat{x}_{1}
\end{array}\right.
$$

where $e=y-\hat{y}=x_{1}-\hat{x}_{1}$ and $\hat{x}_{1}, \hat{x}_{2}, \hat{x}_{3}$ is the observer of $x_{1}$, $x_{2}, x_{3} .0<\alpha_{1}<1,0<\alpha_{2}<1, \sigma_{1}>0, \sigma_{2}>0, l_{i}>0$, $i=1,2,3$ are parameters of observer (6). And the nonlinear function $\mathrm{fal}(\cdot)$ is defined as

$\operatorname{fal}(\epsilon, \alpha, \sigma)= \begin{cases}|\epsilon|^{\alpha} \operatorname{sgn}(\epsilon), & |\epsilon|>\sigma \\ \frac{\epsilon}{\sigma^{1-\alpha}}, & |\epsilon| \leq \sigma\end{cases}$

In generally, $\sigma$ is selected as $\sigma=5-10 T$, where $T$ is sampling period of control. Until now, there is no reliable theoretical analysis method available for third-order ESO. Fortunately [16], if suitable parameters of observer (6) are selected, the following results can be obtained.

$$
\begin{aligned}
& \lim _{t \rightarrow \infty}\left|\tilde{x}_{2}\right|<l_{1}\left(\frac{r}{l_{3}}\right)^{1 / \alpha_{2}}=\varepsilon_{x_{2}} \\
& \lim _{t \rightarrow \infty}\left|\tilde{x}_{3}\right|<l_{2}\left(\frac{r}{l_{3}}\right)^{1 / \alpha_{2}}=\varepsilon_{f(x)}
\end{aligned}
$$

where $\tilde{x}_{2}=x_{2}-\hat{x}_{2}, \tilde{x}_{3}=x_{3}-\hat{x}_{3}$. Hence, we know the suitable observer parameters can make the state estimation errors $\tilde{x}_{1}, \tilde{x}_{2}$ and function estimation error $\tilde{f}(x)=\tilde{x}_{3}=\hat{f}(x)-f(x)$ are uniformly ultimately bounded.

Observer (6) is given the following form as

$$
\left\{\begin{array}{l}
\dot{\hat{x}}_{1}=\hat{x}_{2}+\eta_{1} \\
\dot{\hat{x}}_{2}=\mathrm{b} u+\eta_{2}
\end{array}\right.
$$

where $\eta_{1}=-l_{1} e, \eta_{2}=\hat{f}(x)-l_{2}$ fal $\left(e, \alpha_{1}, \sigma\right)$, and $\mathrm{b}=1$.

\subsection{Backstepping Controller Design}

Based on the dynamic model (9), we can define the state tracking errors $e_{1}$ and $e_{2}$ as follows:

$e_{1}=\hat{x}_{1}-x_{1}^{d}, e_{2}=\hat{x}_{2}-\hat{x}_{2}^{d}$

where $x_{1}^{d}$ and $\hat{x}_{2}^{d}$ are the references of $\hat{x}_{1}$ and $\hat{x}_{2}$, respectively. Note that $x_{1}^{d}$ is given by command reference and $\hat{x}_{2}^{d}$ will be designed later.
From (10), we obtain the the derivatives of $e_{1}$ and $e_{2}$ as

$$
\begin{aligned}
& \dot{e}_{1}=\hat{x}_{2}+\eta_{1}-\dot{x}_{1}^{d} \\
& \dot{e}_{2}=\mathrm{b} u+\eta_{2}-\dot{\hat{x}}_{2}^{d}
\end{aligned}
$$

Step 1: Let us define $\hat{x}_{2}$ is the virtual control for $\mathrm{x} 1$ subsystem, and choose $\hat{x}_{2}^{d}=\hat{x}_{2}$ as the ideal control input. It is remarked that, in the step, the task is to stabilize the dynamic (11) under respect to Lyapunov function $V_{1}=\frac{1}{2} e_{1}^{2}$.

The time derivative of $V_{1}$ is

$\dot{V}_{1}=e_{1}\left(\hat{x}_{2}+\eta_{1}-\dot{x}_{1}^{d}\right)$

The virtual controller (i.e., outer-loop controller) can be designed as

$\hat{x}_{2}^{d}=\dot{x}_{1}^{d}-\eta_{1}-c_{1} e_{1}$

where $c_{1}>0$ is a designed control gain of out-loop. Substituting (14) into (13), we have $V_{1} \leq 0$.

From (12) and (14), we define the Lyapunov function $V_{2}=\frac{1}{2} e_{1}^{2}+\frac{1}{2} e_{2}^{2}$

Then, taking the time derivative of $V_{2}$ yields

$$
\begin{aligned}
\dot{V}_{2} & =e_{1} \dot{e}_{1}+e_{2} \dot{e}_{2}=-e_{1}\left(c_{1} e_{1}+\hat{x}_{2}-\hat{x}_{2}^{d}\right)+e_{2} \dot{e}_{2} \\
& =-c_{1} e_{1}^{2}+e_{1} e_{2}+e_{2} \dot{e}_{2}
\end{aligned}
$$

Designing $\theta=\eta_{2}-\frac{\partial \hat{x}_{2}^{d}}{\partial \hat{x}_{1}}\left(\hat{x}_{2}+\eta_{1}\right)-c_{1} \dot{x}_{1}^{d}-\ddot{x}_{1}^{d}$, and substituting (12) yields

$$
\begin{aligned}
\dot{V}_{2} & =e_{1} \dot{e}_{1}+e_{2} \dot{e}_{2} \\
& =-c_{1} e_{1}^{2}+e_{2}\left[e_{1}+\mathrm{b} u+\eta_{2}-\frac{\partial \hat{x}_{2}^{d}}{\partial \hat{x}_{1}}\left(\hat{x}_{2}+\eta_{1}\right)-c_{1} \dot{x}_{1}^{d}-\ddot{x}_{1}^{d}\right]
\end{aligned}
$$

then the global control algorithm is designed as

$$
u=\mathrm{b}^{-1}\left(-c_{2} e_{2}-e_{1}-\theta\right)
$$

where $c_{2}$ is a positive constant to be designed. Then, the time derivative of Lyapunov function $V_{2}$ is described as

$\dot{V}_{2}=-c_{1} e_{1}^{2}-c_{2} e_{2}^{2} \leq 0$

From (16), we can ensure that the closed-loop under global controller (15) is stable. Thus, the designed backstepping controller is effectiveness. 


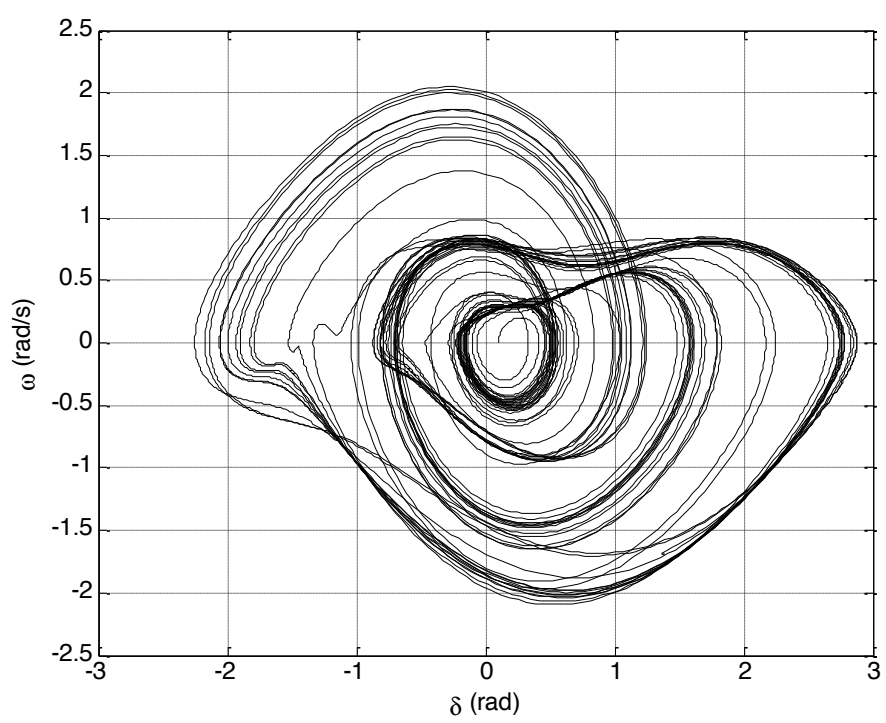

Fig. (2). Chaotic attractor under $\mu=1.3$.
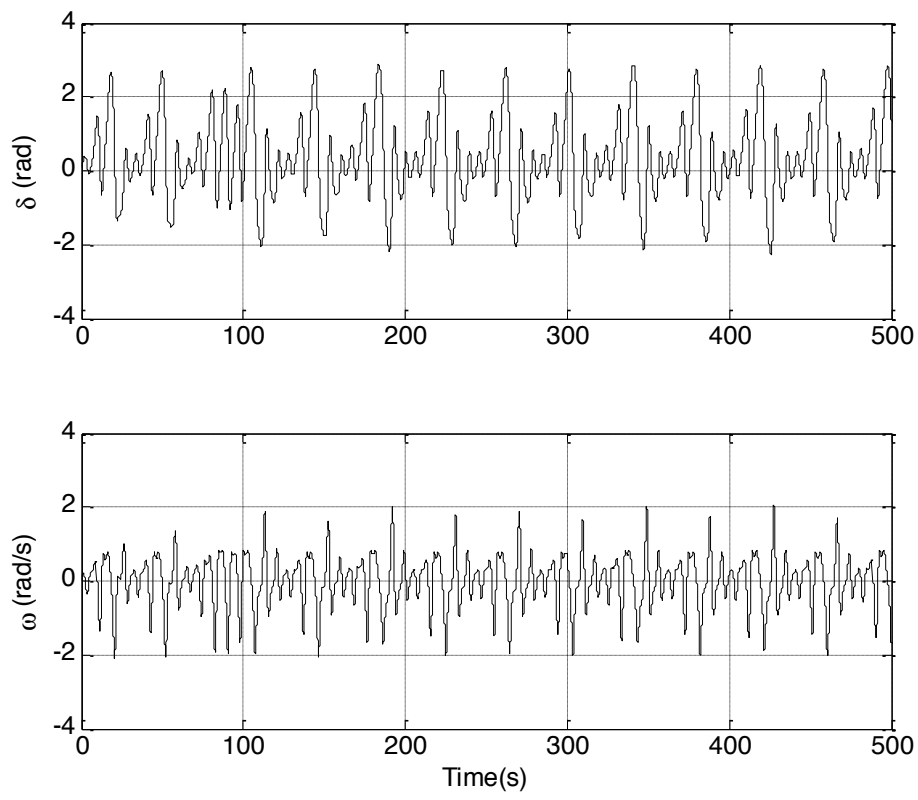

Fig. (3). Timing diagram of power angle $\delta$ and relative power angle velocity $\omega$.

\section{SIMULATION RESULTS}

Simulations were performed in the MATLAB/SIMULINK environment. From numerical analysis of the marine power system's chaotic motion, we can obtain the results that when the amplitude $\mu=1.3$, the marine power system will appear the chaos under $\lambda=0.4$, $\rho=0.2$, disturbance frequency $\gamma=0.8$.

We can obtain the motion state of the marine power system in Figs. (2 and 3). Form Fig. (2), it can be seen that the system power angle and the angular velocity of the phase diagram of movement is periodicity, which shows that the system appeared in chaos. The system experiences a similar random, but does not attenuate oscillations, further validates this point produced a chaotic system.

The parameters of the backstepping controller are chosen as, $c_{1}=c_{2}=2$. The parameters of ESO are designed as $\alpha_{1}=\alpha_{2}=0.9, \sigma_{1}=100, \sigma_{2}=1000, l_{1}=10, l_{2}=100$, $l_{3}=1000$. 


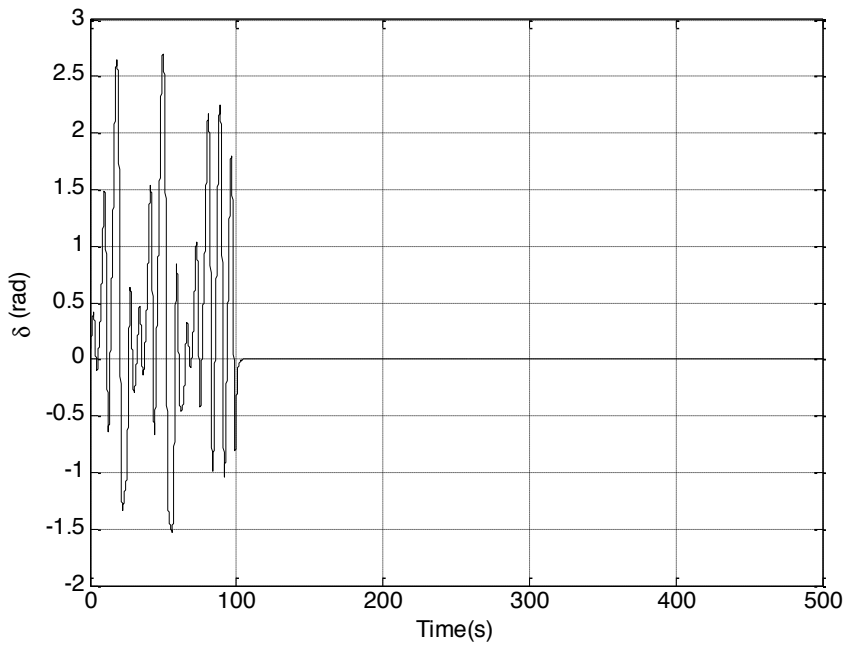

Fig. (4). The curve of power angle $\delta$.

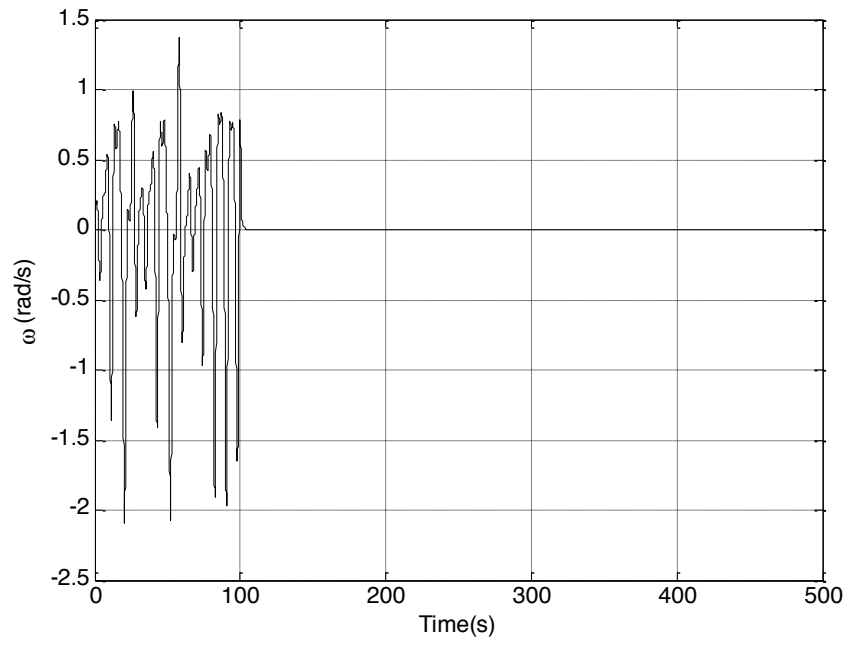

Fig. (5). The curve of power angle $\omega$.

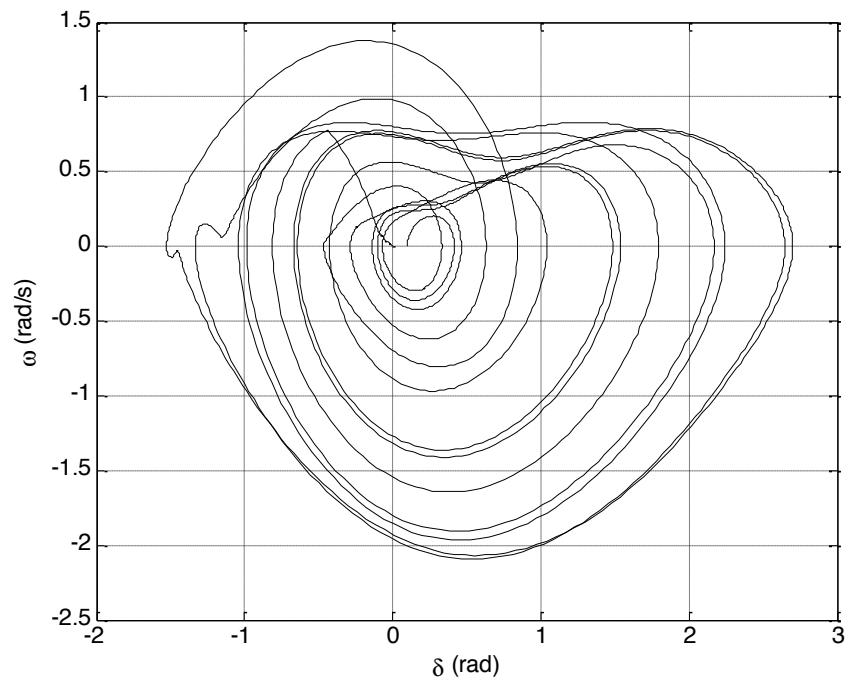

Fig. (6). Phase diagram of power angle $\delta$ and relative power angle velocity $\omega$. 


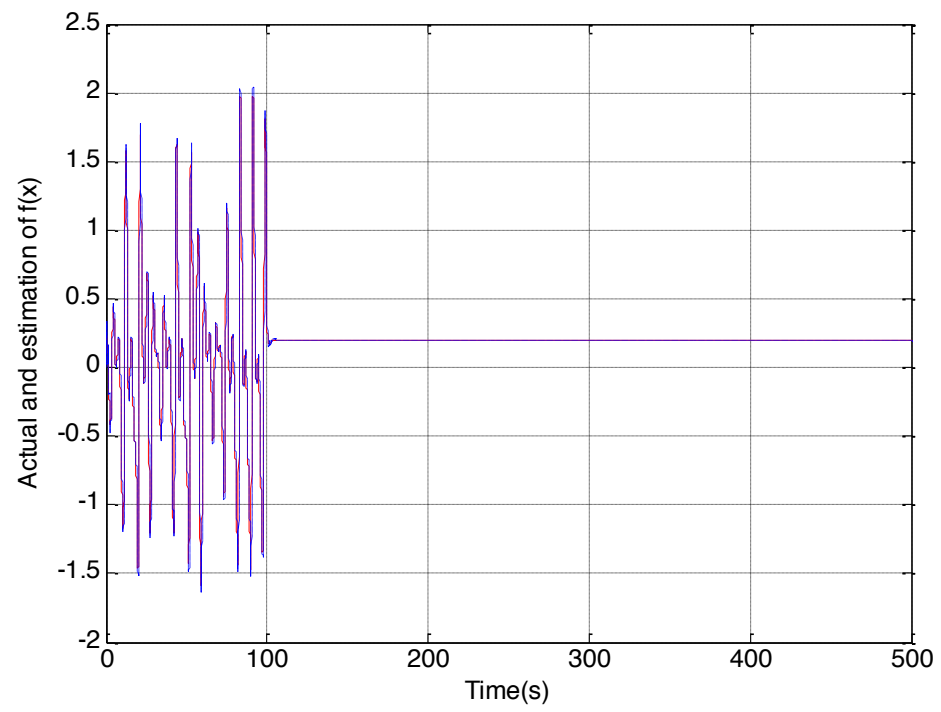

Fig. (7). Actual $f(x)$ function and its estimation $\hat{f}(x)$.

The initial of states is $x_{0}=[0.1,0]^{T}$. In the following simulation, we add the control signal $u$ to the marine power system when the chaotic motion is occurred after 100 seconds. Fig. (4) and Fig. (5) show the curve of power angle and the angular velocity of marine power system with ESO method. And the phase diagram is shown in Fig. (6). Fig. (7) shows the actual $f(x)$ function and the estimation $\hat{f}(x)$.

It can be seen the results from Fig. (5) and Fig. (6), before 100 seconds, power angle $\delta$ and relative power angle velocity $\omega$ are in a chaotic state. While the designed controller is added after 100 seconds, system is quickly stabilized, this indicates the proposed ESO-based control algorithm has a very reliable stabilization ability for the marine power system's chaotic motion.

\section{CONCLUSION}

To rely on observer techniques, we propose a novel model free backstepping control methods for marine power systems. In the developed two model free backstepping controls, there are three main problems are solved, they are 1): Velocity signal does not need to be known. The proposed control algorithms can achieve the close-loop stability without speed sensor. 2): The proposed control methods don't need dynamic mathematical model of marine power systems. $3)$ : The proposed control method can eliminate the impact of derivative signal and control saturation. In addition, Stability analysis is given for closed-loop control system. Simulation results show that the proposed method not only guarantees closed-loop stability of the controlled marine power system, but also identifies well the velocity state and unknown dynamic model.

\section{CONFLICT OF INTEREST}

The author confirms that this article content has no conflict of interest.

\section{ACKNOWLEDGEMENTS}

This work was supported in part by the Fundamental Research Funds for the Central Universities (JUSRP11562).

\section{REFERENCES}

[1] S. Elghali, M. Benbouzid, and J. Charpentier, "Modeling and control of a marine current turbine driven doubly-fed induction generator", IET Renewable Power Generation, vol. 4, no. 1, pp. 1-11, 2010.

[2] H.K. Chen, T.N. Lin, J.H. Chen, "Dynamic analysis controlling chaos and chaotification of a SMIB power system," Chaos Solitons \& Fractals, vol. 24, no. 5, pp. 1307-1315, 2005.

[3] X. Li, and C.A. Caizares, "Chaotic behavior observations in a power system model", IEEE Bucharest PowerTech, Romania, 2009, pp. 40-44.

[4] G. Chen, "Control and anti-control of chaos", Journal of Guangxi Normal University, vol. 20, no. 1, pp. 1-5, 2002.

[5] S. Boccaletti, C. Grebogi, Y. C. Lai, H. Mancini, and D. Maza, "The control of chaos: theory and applications", Physics Reports, vol. 329, no. 3, pp. 103-197, 2000.

[6] C.C. Hwang, J.Y. Hsieh, and R.S. Lin, "A linear continuous feedback control of Chua's circuit", Chaos Solitons \& Fractals, vol. 8, no. 9, pp. 1507-1515, 1997.

[7] M. Feki, "An adaptive feedback control of linearizable chaotic systems", Chaos Solitons \& Fractals, vol. 15, no. 5, pp. 883-890, 2003.

[8] Z.S. Hou and Z. Wang, "From model-based control to data-driven control: Survey, classification and perspective", Information Sciences, vol. 235, pp. 3-35, 2013.

[9] J. Farrell, M. Polycarpou, and M. Sharma, "Longitudinal flight path control using online function approximation", Journal of Guidance, Control Dynamics, vol. 26, no. 6, pp. 885-897, 2003.

[10] D. Swaroop, J. Hedrick, P. Yip, and J. Gerdes. "Dynamic surface control for a class of nonlinear systems", IEEE Transactions on Automatic Control, vol. 45, no. 10, pp. 1893-1899, 2000.

[11] M. Zhang, L. Yin, and L. Qiao, "Adaptive fault tolerant attitude control for cube satellite in low earth orbit based on dynamic neural network", International Journal of Innovative Computing, Information and Control, vol. 10, no. 5, pp. 1843-1852, 2014.

[12] N. Chen, F. Song, G. Li, X. Sun, and C. Ai, "An adaptive sliding mode backstepping control for the mobile manipulator with nonholonomic constraints", Communications in Nonlinear Science Numerical Simulation, vol. 18, no. 10, pp. 2885-2899, 2013.

[13] Y. Xia, P. Shi, G. P. Liu, and D. Rees, "Active disturbance rejection control for uncertain multivariable systems with time delay", 
IET Control Theory and Applications, vol. 1, no. 1, pp. 75-81, 2007.

[14] D.Z. Xu, B. Jiang, and P. Shi, "A novel model free adaptive control design for multivariable industrial processes". IEEE Transactions on Industrial Electronics, vol. 61, no. 11, pp. 6391-6398, 2014.

[15] D.Z. Xu, B. Jiang, and P. Shi, "Adaptive observer based datadriven control for nonlinear discrete-time processes", IEEE Trans- actions on Automation Science and Engineering, vol. 11, no. 4, pp. 1037-1045, 2014.

[16] J.Q. Han, "From PID to active disturbance rejection control", IEEE Transactions on Industrial Electronics, vol. 56, no. 3, pp. 900-906, 2009.

Received: September 16, 2014

Revised: December 23, 2014

Accepted: December 31, 2014

(C) Jianlong et al.; Licensee Bentham Open.

This is an open access article licensed under the terms of the Creative Commons Attribution Non-Commercial License (http://creativecommons.org/licenses/by$\mathrm{nc} / 3.0 /$ ) which permits unrestricted, non-commercial use, distribution and reproduction in any medium, provided the work is properly cited. 